\title{
Disruption Emergency Optimization Model for Transnational Supply Chain
}

\author{
Chunling Liu ${ }^{\mathrm{a}}$, Junfeng Wang ${ }^{\mathrm{b}}$, and Yangjie Tian ${ }^{\mathrm{c}}$ \\ School of Mechanics Engineering and Automation, Wuhan Textile University, Wuhan, China, \\ a chunringliu5@aliyun.com, bJunf_wang@126.com, ${ }^{\mathrm{C}}$ Yj_tian@qq.163.com
}

Keywords: Transnational supply chain; Inventory optimization; Production scheduling; Model predictive control; Disruption

\begin{abstract}
In Supply Chain Management (SCM) due to fluctuations in demand and the "bullwhip effect", customer satisfaction and production inventory cost are difficult to coordinate. So a comprehensive optimization method for the research of supply chain inventory and production scheduling is developed based on multiple model predictive control (MMPC) strategy which has successfully applied to industrial process, and the corresponding control optimization system is constructed, meanwhile made the simulation and analysis in the case of transnational supply chains. The results show that based on MMPC supply chain production and inventory optimization decision-making mechanism can effectively curb the "bullwhip effect", while suppressing large fluctuations in inventory at the same time, to achieve better customer satisfaction.
\end{abstract}

\section{Introduction}

Transnational supply chain system becomes more and more complicated, and the external market demand uncertainty and potential risks may lead to supply chain disruptions, which will affect normal operation of cluster supply chain network. These potential risks include natural disasters, major public health events, and so on. Such as the 2000 Ericsson enterprise, due to the firing of the chip supplier production workshop by lightning, the supply chain was disrupted for more than a month, so Ericsson got the loss of $\$ 2.34$ billion for market shares and order, price of stock fell nearly $13.5 \%$, eventually forcing him out of the mobile phone market; on the 2011 Tsunami in Japan, the supply chains of TOYOTA, Nissan and other Japanese car enterprises have been interrupted, the production suffered a great impact, therefore visible interrupt effect on supply chain is huge.

The model predictive control (MPC) method has the function of rolling optimization, which can respond effectively to uncertainties such as model adaptation, distortion and external disturbance in time, and has a relatively stable dynamic performance. Some literatures involved on multi-period inventory control. Wang \& Daniel \& Rivera (2006) regarded the semiconductor manufacturing industry as the example, using the MPC method to analyze different strategies of supply chain control; the domestic scholar Chen Wenbo (2008) put forward an optimal control method for supply chain network based on MPC. In the light of complex cases in supply chain, some scholars promoted new operation strategies to control the multi-supply-chain system by introducing model switching method. But the existing literatures rarely considered switching model predictive control (MMPC) applied to multiple supply chains, especially in the analysis of supply chain disruptions.

According to the cross-chain emergency complementary situation between chain and chain, modeling analysis and solution are discussed for emergency management of cluster supply chains by application of multi model predictive control (MMPC) method in this paper.

\section{Modeling on supply chain control}

Consider two supply chains within an industrial cluster with transnational firms, each including a supplier, manufacturer, and distributor. Suppose that two chains produce homogeneous products, in which a chain is interrupted, and another strand can provide supplement when its own inventory is sufficient. In order to control the multi-chain nodes in time, the inventory level of each node is 
taken as the control object, and the market demand is taken as the feedback, as shown in figure 1.

Assume that the cluster supply chain system produce a single product, $I(t)$ is inventory associated with the time $t, \mathrm{u}(\mathrm{t})$ is order quantity of supplier at t moment, and the goods will have a certain loss after a certain time $\theta_{11}$ of transportation and assembly, so that the loss ratio coefficient is $\mathrm{K}$, the output of supplier 1 for the downstream node is $\mathrm{d}(\mathrm{t})$, by these known parameters, the material balance equation (Eq.1)of the first nodes (supplier 1)can be set up in the supply chain 1 .

$\frac{d I_{11}(t)}{d t}=K_{11} u_{11}\left(t-\theta_{11}\right)-d_{21}(t)$

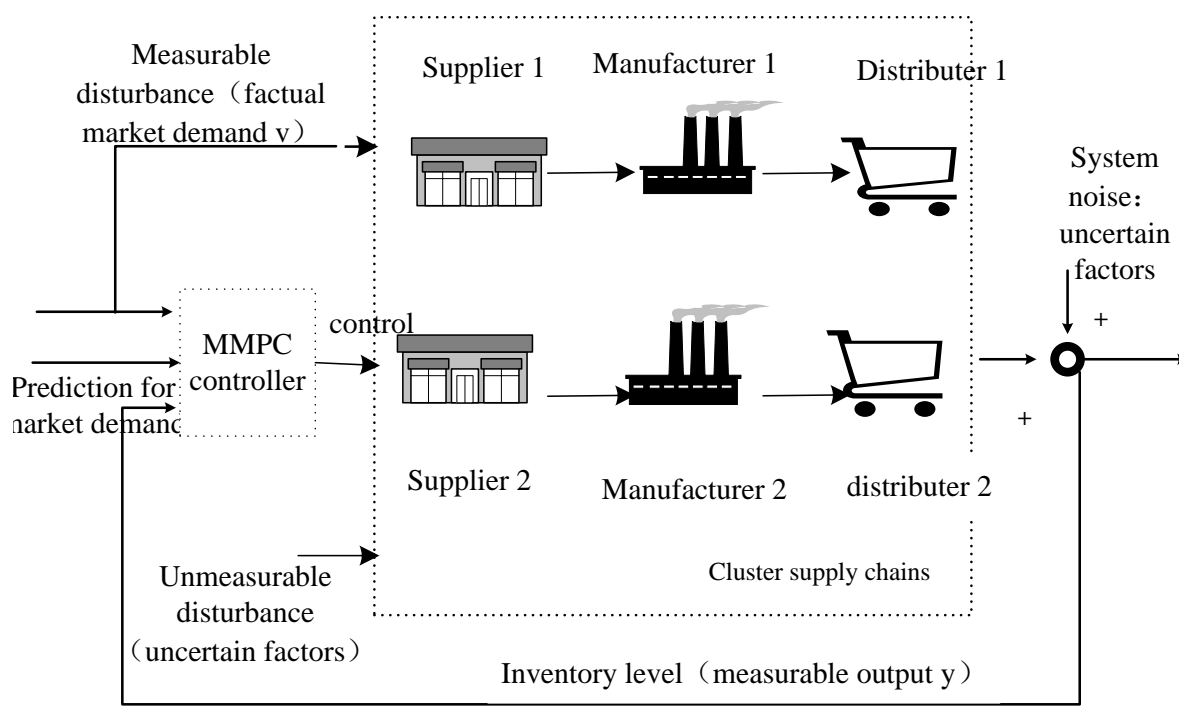

Figure 1. Structure of cluster supply chains based on MMPC

Where, $i j$ is the $i$ node in $j$ chain (supplier, $=1$, manufacturer, $=2$, seller, $=3$ );

$K_{i j}$ is the loss coefficient of the $i$ node in $j$ chain;

$I_{i j}(s)$ is the inventory of the $i$ node in $j$ chain at time T;

$t$ - Discrete time point; $\theta_{i j}$ - the delay of the $i$ node in $j$ chain to the downstream system

$u_{i j}(s)$ - the quantity of the $i$ node in $j$ chain at the t moment;

$d_{f_{j}}(s)$-Mmarket demand for sellers in $j$ chain at t moment;

$\left(\begin{array}{c}I_{11}(s) \\ I_{21}(s) \\ I_{31}(s) \\ I_{12}(s) \\ I_{22}(s) \\ I_{32}(s)\end{array}\right)=\left(\begin{array}{cccccc}\frac{K_{11} e^{-\theta_{1 s} s}}{s} & -\frac{1}{s} & 0 & 0 & 0 & 0 \\ 0 & \frac{K_{21} e^{-\theta_{21} s}}{s} & -\frac{1}{s} & 0 & 0 & 0 \\ 0 & 0 & \frac{K_{31} e^{-\theta_{31 s}}}{s} & 0 & 0 & 0 \\ 0 & 0 & 0 & \frac{K_{12} e^{-\theta_{25} s}}{s} & -\frac{1}{s} & \\ 0 & 0 & 0 & 0 & \frac{K_{22} e^{-\theta_{22} s}}{s} & -\frac{1}{s} \\ 0 & 0 & 0 & 0 & 0 & \frac{K_{32} e^{-\theta_{25} s}}{s}\end{array}\right)\left(\begin{array}{c}u_{11}(s) \\ u_{21}(s) \\ u_{31}(s) \\ u_{12}(s) \\ u_{22}(s) \\ u_{32}(s)\end{array}\right)-\ldots .\left(\begin{array}{cc}0 & 0 \\ 0 & 0 \\ 0 & 0 \\ 0 & 0 \\ -\frac{1}{s} & 0 \\ 0 & -\frac{1}{s}\end{array}\right)\left(\begin{array}{l}d_{f 1}(s) \\ d_{f 2}(s)\end{array}\right)$

In order to get better control performance of the whole system, a model of cluster supply chain system based on deviation is established on the basis of the above model. The deviation is designed as follows: $\bar{I}_{i j}$ - the benchmark inventory quantity of the $i$ node in $j$ chain (a constant set by the decision maker); $\hat{I}_{i j}$ - the inventory deviation of the $i$ node in $j$ chain; $\bar{u}_{i j}$-The order benchmark quantity (or self production) of $i$ node for the upstream node in the supply chain $j$ (which is a constant set by the decision maker); $\hat{u}_{i j}$-The order deviation (or self production) of $i$ node for the upstream node in the supply chain $j$. 


\section{Determination of Objective Function}

In above case, system model of the deviation based on MPC is established for the cluster supply chain, in order to fully prevent supply chain disruption risks, on the other hand, to meet the needs of the market and reduce inventory of the cluster supply chain itself, so the objective function of the model may be deduced.

The following constraints should be met (refer with Eq.3)

$$
\begin{aligned}
& \hat{u}_{j_{\min }}(k+i) \leq \hat{u}_{j}(k+i \mid k) \leq \hat{u}_{j_{\max }}(k+i) \\
& \Delta u_{\min }(k+i) \leq \Delta u(k+i \mid k) \leq \Delta u_{\max }(k+i) \\
& \hat{I}_{j_{\min }}(k+i) \leq \hat{I}_{j}(k+i \mid k) \leq \hat{I}_{j_{\max }}(k+i)
\end{aligned}
$$

Where $\hat{u}_{j} 、 \Delta u 、 \hat{I}_{j}$ are the parameter matrixes corresponding to each node.

Among them, the objective function is extended as

$$
\begin{aligned}
& J=\sum_{i=0}^{P-1}\left(\sum_{j=1}^{n_{t}}\left|\omega_{i+1, j}^{I}\left(\hat{I}_{j}(k+i+1 \mid k)-r_{j}(k+i+1)\right)\right|^{2}\right)+\sum_{j=1}^{n_{i}}\left|\omega_{i, j}^{\Delta u} \Delta u_{j}(k+i \mid k)\right|^{2}+\sum_{j=1}^{n_{u}}\left|\omega_{i, j}^{u}\left(\hat{u}_{j}(k+i \mid k)-u_{j \mathrm{target}}(k+i)\right)\right|^{2} \\
& \text { Where } r_{j}(k+1), \quad u_{j \mathrm{targ} e t}(k+i) \text { are reference output and input sequence. }
\end{aligned}
$$

\section{Simulation of Emergency Response Strategy for Supply Chain Disruption Based on MPC}

The daily operation of the supply chain is applicable basis of MPC in the optimization of supply chain management, and whether responds to interrupt timely and effectively, and makes the whole supply chain can operate normally during interruption, which are the great role of MPC should play.

In the following, this paper will use Matlab to simulate the interruption cases mentioned in the previous chapter, and try to verify the robustness of the proposed supply chain operation by simulation.

Consider all the constant constraints in the model that normally operate in the above sections. Consider the following interrupt model (refer with Fig. 2):

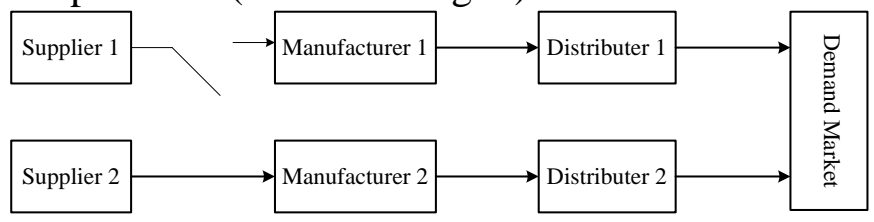

Figure 2 case of supplier interruption

Therefore, we use the mentioned "emergency supply strategy", which is to conclude a supply agreement under a state of emergency between the two supply chains that are close but not overlapping: when interrupt on supplier 1 occurs, the supplier 2 will provide emergency supplies for manufacturers 1 , and vice versa. The deployment process may be operated by MPC, which reduces the cost of the purchase, on the other hand, reduces the risk of interruption caused by a single supplier.

Set benchmark inventory for suppliers, manufacturers and distributors are 400, 300, and 300. We assume that the maximum warehouse capacity is 600 , and that in each simulation cycle transport capacity in transit has no more than 400 .

The simulation results are as follows:

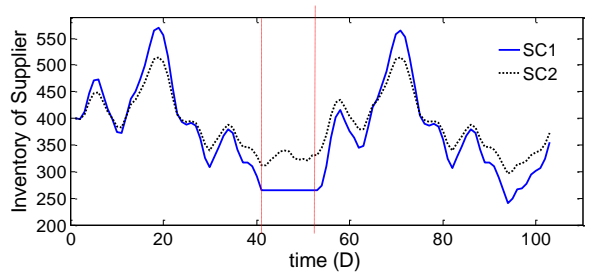

(a)Inventory of supplier

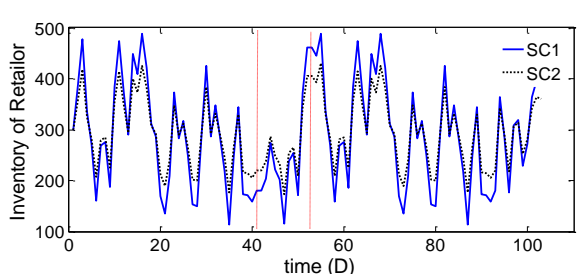

(b) Inventory

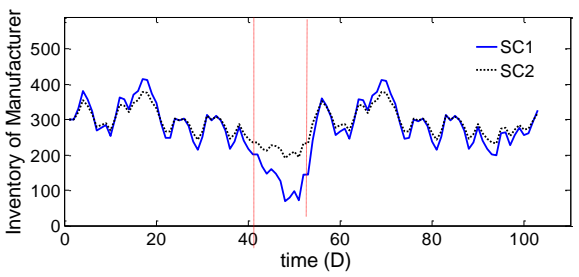

of manufacturer (c) Inventory of distributer

Figure 3 inventory of nodes in supply chain system 
In the simulation results above, the red dotted line is exactly the process of the whole interruption. As you can see, the system first in the initial state run 40 cycles, throughout this period of operation, inventory operates firmly around the baseline, and the two dealers are also very good to meet the needs of the market, the supply chain is highly efficient and orderly.

Then, in some cases, the supplier 1 encountered the impact of breaking into it and could no longer participate in the operation of the entire node. In other words, the supplier 1 was interrupted. As you can see, in the interruption time, stock of the vendor 1 is frozen and at the same time it stops all operations itself. The MMPC controller detects the interruption, and then switch to the interrupt model, each node in the contract by the controller's command, coordinate together to help supply chain 1 after supplier 1 break into disruptions, to ensure that it will not cause too much damage because of interruption. Thus, the influence of the interrupt is weakened due to being passing to other nodes by the controller. As shown in figures, in order to keep inventory benchmark and eliminate the interrupt effects of supplier 1, in interrupt process, operation plan data of each node (freight) fluctuates obviously become intense. Because the market demand is satisfied, from inventory of manufacturers 1 we can see an obvious decline in figure (3), through that the expected goal of inventory and multi-node freight with constrained control has been reached well.

\section{Conclusion}

Through the research of MMPC, the robustness and stability of the control method are introduced into the management of the supply chain system. The model switching algorithm is used to improve the MPC algorithm in the industrial field, which makes the control algorithm more suitable for the diversity of the supply chain control. At the same time, regarding with the common problems for the interruption of supply chain system, the framework and protocol of the emergency response are put forward about two supply chains and even multiple supply chains, so that partners in the two supply chains overcome the interruption through cooperation, reduce losses, to achieve mutual benefit and win-win.

\section{Acknowledgment}

The paper is supported in part by China NASFC (National Science Function Committee) ( 71472143), This research is also supported by China Education Ministry (15YJA630035).

\section{References}

[1] Tomlin B.. On the value of mitigation and contingency strategies for managing supply chain disruption risks. Management Science, 2006, 52(5):639-657

[2] Liu Xilong, Ji Jianhua. Study on Design of flexible supply chain network based on emergency supply. Control and decision, 2007, 22 (11): 1223-1227

[3] Yu Hui, Chen Jian, Yu Gang. How to coordinate supply chain to deal with emergencies. System engineering theory and practice, 2005, 25 (7): 9-16

[4] Kothare M V, Balakrishnan V, Morari M. Robust constrained model predictive control using linear matrix inequalities, Automatica, 1996, 32: 1361-1379.

[5] Kapsiotis G, and Tzafestas S, Decision making for inventory/production planning using model-based predictive control. In Parallel and Distributed Computing in Engineering Systems, edited by S. Tzafestas, P. Borne and L. Grandinetti, pp. 551-556, 1992 (Elsevier : Amsterdam)

[6] Wenlin Wang, et al. Model predictive control strategies for supply chain management in semiconductor manufacturing. Int. J. Production Economics, 107(2006), 56-77. 